\title{
Interferometric Noise Characterisation of a 2-D Time Spreading Wavelength Hopping OCDMA Networks using FBG Encoding/Decoding
}

\author{
Craig Michie $^{1}$, Robert Atkinson ${ }^{1}$, Ivan Andonovic ${ }^{1}$, Ivan Glesk $^{2}$, Paul Prucnal $^{2}$ \\ Kensuke Sasaki ${ }^{3}$, Gyaneshwar Gupta ${ }^{3}$ \\ ${ }^{2}$ Department of Electronic and Electrical Engineering, University of Strathclyde, Glasgow, Scotland G1 $1 X W$ \\ ${ }^{2}$ Department of Electrical Engineering, Princeton University, Princeton, NJ 08544 \\ ${ }^{3}$ Oki Electric Industry Co., Ltd., Advanced Devices Laboratories \\ Email: c.michie@eee.strath.ac.uk
}

\begin{abstract}
The results of a range of experimental characterization exercises of interferometric noise (IN) for the case of a representative 2-D time spreading wavelength-hopping (2-D TW) optical code family are presented. Interferometric noise is evaluated at a data rate of $2.5 \mathrm{Gbps}$ within an OCDMA network emulation test bed established utilising fibre Bragg grating encoders/decoders. The results demonstrate that this form of noise introduces significant system power penalties and must be taken into consideration in any OCDMA network designs and implementations.
\end{abstract}

Keywords: optical code-division multiple access (OCDMA), time-wavelength optical codes, beat noise, fibre Bragg grating coders.

\section{INTRODUCTION}

Optical code-division multiple access (OCDMA) is a system approach able to support bursty, variable data rate traffic with a reduced network management overhead. The performance of OCDMA systems at the physical level is limited by a combination of noise sources, the more critical being multiple access interference (MAI) and interferometric noise (IN) [1-3]. Most optical systems are prone to IN viz. wavelength division multiplexing (WDM) [4-5], time division multiplexing (TDM) [6-7], sub-carrier multiplexing [8-9] and OCDMA [10-12].

Most of the literature has studied the performance of 2-D TW scheme under the MAI limited case. However, for a more accurate system evaluation as indicated by many other all-optical network analyses, interferometric noise must be taken into consideration. There are different sources of unwanted crosstalk signals including cochannel interference (MAI), optical component imperfections, multiple reflections or fibre nonlinearity. This paper presents results based on the experimental characterisation of beat noise due to the homodyne beating arising from co-channel interference between 2-D TW codes, and forms the basis of a framework that may be extended to consider other impairments.

\section{EXPERIMENT}

Bit Error Rate (BER) measurements were performed on the OCDMA test bed with Fiber Bragg Gratings (FBG) used for encoding and decoding the data. The network was designed to support up to four users (four different encoders) sending data and one receiver, comprising a decoder which was matched to one of the encoders (users). Different communication scenarios were emulated by varying number of users and by adjusting the individual properties e.g. polarization or delay of the transmitted user signals. For each scenario, BER measurements were taken in order to quantify the detrimental effect of interferometric noise on the quality (in terms of power penalty) of the selected channel.

Figure 1 is a schematic of the test bed. The light source was a mode locked super continuum (SC) laser. The $12 \mathrm{~nm}$ flat $\mathrm{SC}$ is generated from the propagation of a $1.6 \mathrm{ps}$ model locked pulse (at a $2.5 \mathrm{GHz}$ repetition rate) from an erbium doped fibre laser through a dispersion decreasing fibre (DDF). Two pulse streams are produced by a $20 / 80$ splitter, the outputs of which were launched into two polarisation insensitive electro-absorption (EA) modulators driven at $2.5 \mathrm{Gbps}$ from two independent bit error testers (BERTs) generating uncorrelated $2^{15}-1$ PRBS sequences.. The two main branches represented the 'data' - modulated signal for the primary encoder (user), designated SPU1 - and the 'interferers' - the second modulator was used to modulate data signal for the remaining encoders (users), designated SPU4, SPU5, and SPU6. After optical amplification through an EDFA, both branch signals were sent to the FBG encoders. 


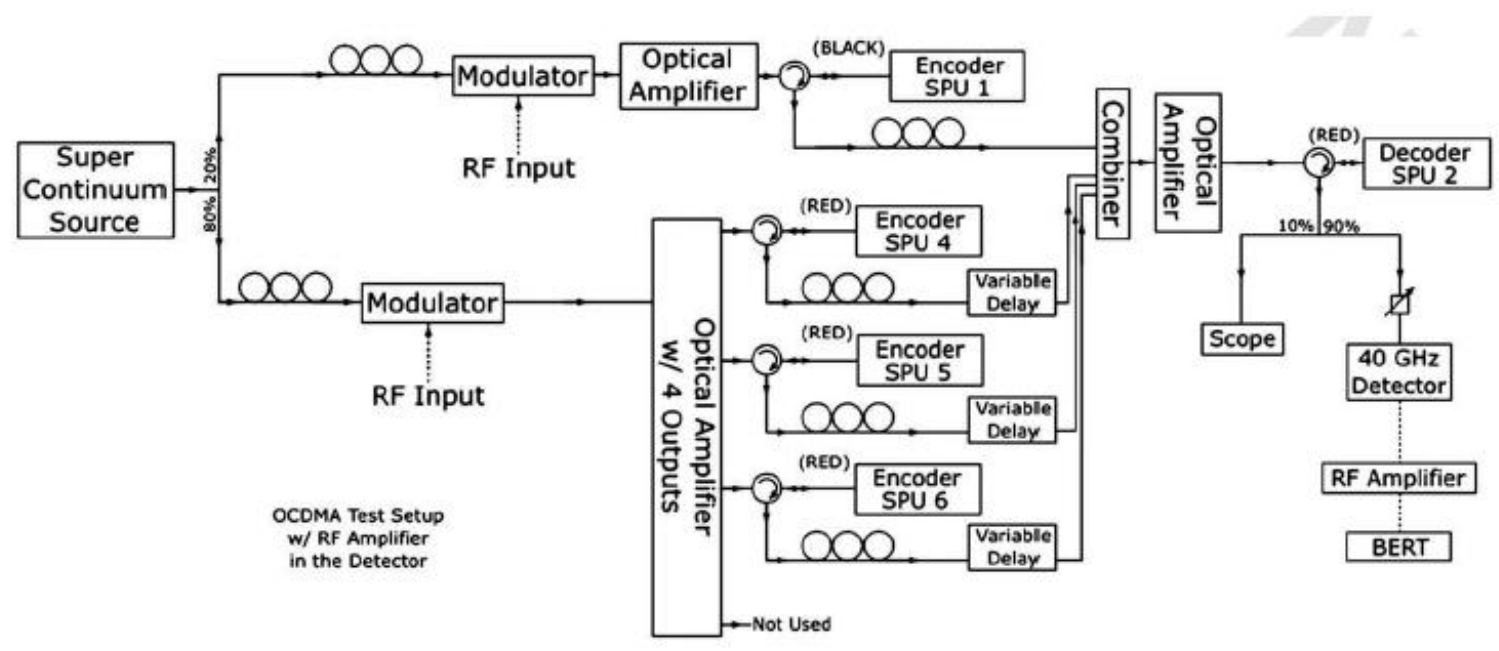

Figure 1. OCDMA networking test bed.

To provide flexibility in the type of network scenarios that the test bed could emulate, each 'interferer' signal (SPU4, SPU5 and SPU6) was passed through a polarization loop controller (PLC) and a tunable optical (manually controlled) delay line (TDL). The TDLs were used to adjust the position of the cross-correlation interfering signals in relation to the desired data represented by the auto-correlation peak. The TDLs also allow for adjustment of delays betw een different users and thus providing the ability to establish various interference conditions. The PLCs control the relative polarization between the cross-correlation and autocorrelation signals, providing the ability to establish the worst case for the generation of beat noise with polarizations aligned and the sum-of-intensities (representative of the more conventional MAI) case with crossed polarizations. After the encoding and conditioning of the signals was performed, all four signals are combined in a $1 \times 4$ optical multiplexer, amplified further by an EDFA and transmitted through a short length of optical fibre. At the receiving end, the combined signals were presented to a decoder, also a FBG device designated as SPU2. The decoder is a mirror image of an encoder to which it is paired i.e. the delays between the reflected wavelengths are inverted, in so doing realigning the wavelengths in the time domain and constructing the auto-correlation peak comprising the incoherent summation of four chips. In this way, signals which are matched with the encoder produce a strong auto-correlation peak, while signals sent with different codes produce crosscorrelations. The decoded signal was sent to a bit-error-tester (BERT) through an attenuator and detector. The signals were also monitored on a digital sampling oscilloscope (Tektronix CSA8000) through a 30GHz frontend detector. The optical attenuator controlled the power of the signal and simulated degrading network conditions.

The chip positions for all four transmitter encoders are summarized in Table 1.

Table 1. Summary of the temporal positions of each chip within the encoders. Chip timeslot is 25ps; code block is $400 \mathrm{ps}$.

\section{Encoders:}

\begin{tabular}{l|c|c|c|c|}
\multicolumn{1}{c}{} & $\lambda_{1}$ & $\lambda_{2}$ & $\lambda_{3}$ & $\lambda_{4}$ \\
\cline { 2 - 5 } SPU1 & 15 & 10 & 5 & 0 \\
\cline { 2 - 5 } SPU4 & 7 & 0 & 13 & 6 \\
\cline { 2 - 5 } SPU & 2 & 11 & 0 & 9 \\
\cline { 2 - 5 } SPU6 & 9 & 6 & 3 & 0 \\
\cline { 2 - 5 } & \multicolumn{5}{c}{ Chip position }
\end{tabular}

Decaders

SPU2

\begin{tabular}{|l|c|c|c|}
$\lambda_{1}$ & $\lambda_{2}$ & $\lambda_{3}$ & $\lambda_{4}$ \\
\hline 0 & 5 & 10 & 15 \\
\hline \multicolumn{4}{|c}{ Chip position }
\end{tabular}

The decoder, SPU2, had the exact gratings as SPU1, but with a reversed order of chip positions. An autocorrelation results for a code match, such as SPU1 and SPU2; conversely a cross-correlation is produced for any mismatch condition e.g. SUP4, SUP5 and SUP6 with SUP2. Since the spread length was 31, the designed code block window (equivalent to the data rate) $400 \mathrm{ps}$, and the code length 16 , the spread length is greater than the code length. Therefore when analyzing the observation window - which is equivalent to the code length - 
overlaps between consecutive chips can occur. For example, the cross correlation generated from SPU4 has chip positions at 7, 5, 23, and 21. In a window length of 16 chips, chips from 7 and 23, 5 and 21, would overlap, thus creating a two double-peak pulses. Eye diagrams for a correlated codes and cross-correlation outputs for SPU4 (left), SPU5 (center) and SPU6 (right) with SUP2 are shown below in Figure 2.

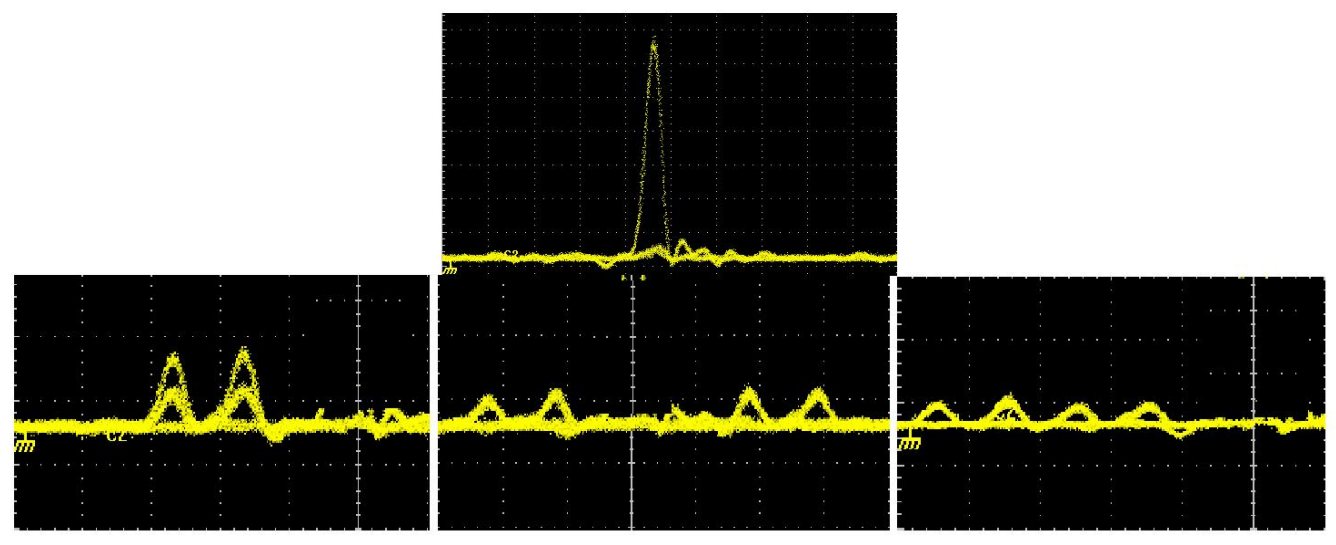

Figure 2. Output eye diagrams from the receiver depicting the autocorrelation (SUP1, above) and three cross correlation codes: SPU4 (left), SPU5 (center) and SPU6 (right). Please note that the cross-correlation for SUP4 was established to be two-chip coincident in order to facilitate the experimentation.

A variety of user interference scenarios were investigated, these will be the subject of a further publication. As an example, Figure 3 below shows BER measurements for SPU1 with two other users present on the channel i.e. an equivalent of two cross-correlation chips directly overlap the auto-correlation peak.

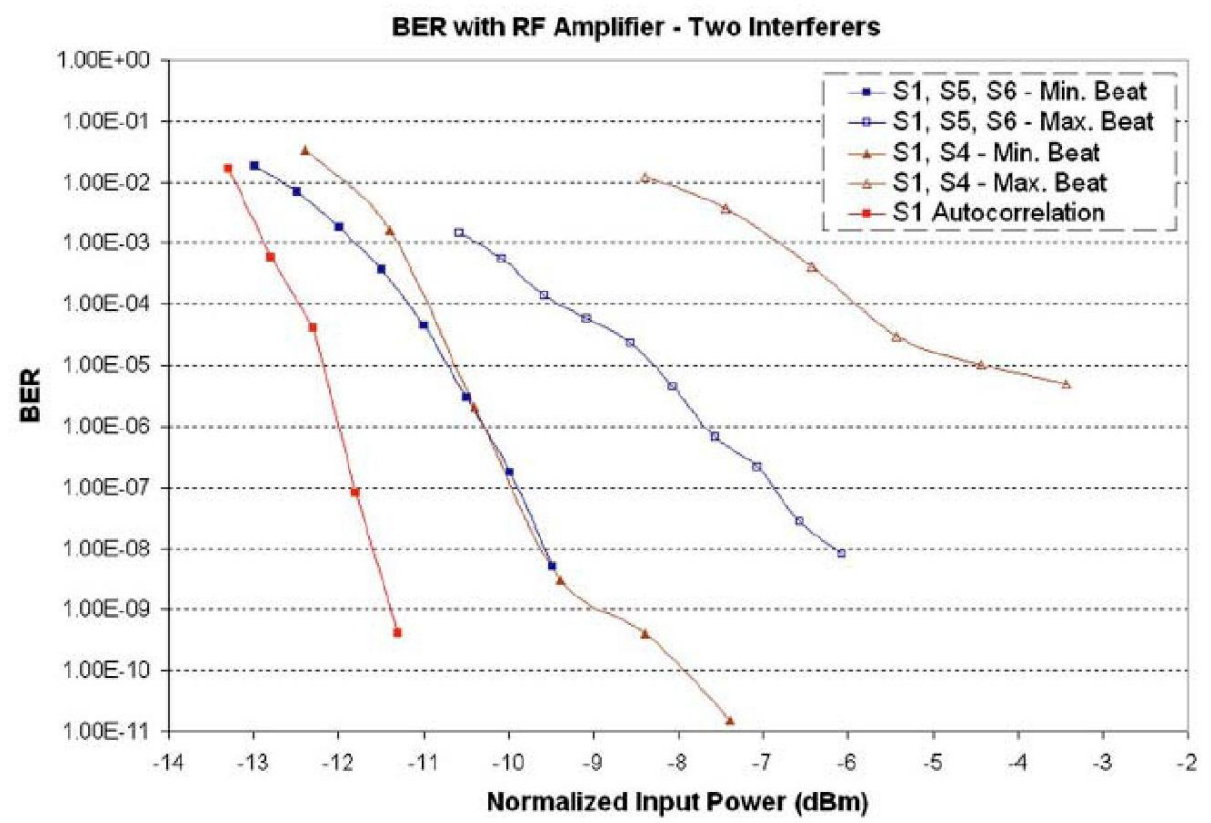

Figure 3. BER as a function of normalized received power for the two interferer scenario, three users on the channel, with two chip overlap of the auto-correlation peak, minimized/maximized levels of beat noise.

\section{RESULTS}

Beat noise was evaluated experimentally at a data rate of $2.5 \mathrm{Gbps}$ for 2-D TW OCDMA codes. A series of BER measurements were undertaken under a range of emulated interference conditions. The measured power penalties represent the worst case of aligned polarizations and complete overlap of the interfering term(s) with the auto-correlation peak. The more practical scenarios that yield the exact power penalties will be a strong function of traffic statistics and network architectures. Nevertheless the results have provided clear experimental evidence that beat noise is indeed a key limiting factor. In summary, within experimental error, the average power penalties as a function of the number of interferers are: 
- one interferer, no overlap; negligible;

- one interferer, with one chip overlap; between $2 \mathrm{~dB}-3 \mathrm{~dB}$ max and $<1 \mathrm{~dB}$ min;

- one interferer, with one chip overlap from one user and no overlap from another; between $2 \mathrm{~dB}-3 \mathrm{~dB}$ max and $<1 \mathrm{~dB}$ min;

- two interferers with two chip overlap (from two different users SPU5, SPU6); $5 \mathrm{~dB}$ max and $1 \mathrm{~dB}$ min;

- two interferers with two chip overlap (from a single SPU4); $7 \mathrm{~dB}$ max and $1 \mathrm{~dB}$ min;

- three interferers with three chip overlap; error floor max and $10 \mathrm{~dB}$ min;

- interferers only; negligible for both max and min.

Thus for a scenario where the interferers are $\sim 3 \mathrm{~dB}$ lower in total power to that of the desired user, results indicate that this form of noise can introduce a significant power penalty at a BER of $10^{-9}$ :

- for a single user with non-overlapping interferers; no significant power penalty,

- for a single interferer, $2 \mathrm{~dB}$ max and $0.5 \mathrm{~dB}$ min;

- for two interferers, $6 \mathrm{~dB}$ max, $2 \mathrm{~dB}$ min;

- for three interferers, error floor and $8 \mathrm{~dB}$ min.

For the case where only the interferers are overlapping; no significant power penalty for both max and min.

\section{CONCLUSIONS}

The experiments reported here represent the beginning of a more extensive evaluation of the impact of a range of noise processes on an OCDMA transmission scheme. Much of the work that has been carried out in this respect has been theoretical and has not been validated experimentally. The data presented here confirms that the level of beat noise generated is a function of co-channel interference power level, which in this case is manifest through an increase in the number of interfering users overlapping with the auto-correlation peak.

\section{ACKNOWLEDGEMENTS}

This research was supported in part by EPSRC overseas travel grant D032547/1.

\section{REFERENCES}

[1] J. O'Reilly, C. Appleton: System performance implications of homodyne beat noise affects in optical fiber network, IEE Proc., vol. 142, pp. 143-148, June 1995.

[2] P.J. Legg, M. Tur, I. Andonovic: Solution paths to limit interferometric noise induced performance degradation in ASK/direct detection lightwave networks, J. Lightwave Technol., vol. 14, pp. 1943-1954, Sept. 1996.

[3] J. Attard, J. Mitchell, C. Rasmussen: Performance analysis of interferometric noise due to unequally powered interferers in optical networks, J. Lightwave Technol., vol. 23, pp. 1692-1703, Apr. 2005.

[4] Y. Shen, K. Lu, W. Gu, Coherent and incoherent crosstalk in WDM optical networks, J. Lightwave Technol., vol. 17, pp. 759-764, May 1999.

[5] T. Kamalakis, T. Spicopoulos: Asymptotic behaviour of in-band crosstalk noise in WDM networks, IEEE Photon. Technol. Lett, vol. 15, pp. 476-478, Mar. 2003.

[6] J. Zhang, et al: Interferometric noise in optical time division multiplexing transmission system, J. Lightwave Technol., vol. 20, pp.1329-1334, Aug. 2002.

[7] P. Legg, et al: Inter-channel crosstalk phenomena in optical time division multiplexed switching networks, IEEE Photon. Technol. Lett., vol. 6, pp. 661-663, May 1994.

[8] C. Desem: Optical interference in lightwave subcarrier multiplexing systems employing multiple optical carriers, Electron. Lett., vol. 24, pp. 50-51, Jan. 1988.

[9] S. Soerensen: Optical beat noise suppression and power equalization in subcarrier multiple access passive optical networks by downstream feedback, J. Lightwave Technol., vol. 18, pp.1337-1347, Oct. 2000.

[10] E. Smith, P. Gough, D. Taylor: Noise limits of optical spectral-encoding CDMA systems, Electron. Lett., vol. 31, pp. 1469-1470, Aug. 1995.

[11] E. Smith, R. Blaikie, D. Taylor: Performance enhancement of spectral-amplitude coding optical CDMA using pulse position modulation, IEEE Trans. Comm., vol. 46, pp.1176-1185, Sept. 1998.

[12] Z. Wei, H. Shalaby, H. Ghafouri-Shiraz; Modified quadratic congruence codes for fiber Bragg grating based spectral amplitude coding optical CDMA systems, J. Lightwave Technol., vol. 19, pp.1274-1281, Sept. 2001. 